DOE/ER/ 60487

\title{
DEVELOPMENT OF MORE EFFICACIOUS TC-99M ORGAN IMAGING AGENTS FOR USE IN NUCLEAR MEDICINE BY ANALYTICAL CHARACTERIZATION OF RADIOPHARMACEUTICALS
}

\author{
Annual Technical Progress Report \\ Sepatember 1, 1992 - August 31,.1993
}

William R. Heineman

Department of Chemistry University of Cincinnati Cincinnati, OH 45221-0172

May 3, 1993

Prepared for

THE DEPARTMENT OF ENERGY

DOE GRANT NO. DOE-FG02-86ER60487

\section{DISCLAIMER}

This report was prepared as an account of work sponsored by an agency of the United States Government. Neither the United States Government nor any agency thereof, nor any of their employees, makes any warranty, express or implied, or assur: es any legal liability or responsibility for the accuracy, completeness, or usefulness of any information, apparatus, product, or process disclosed, or represents that its use would not infringe privately owned rights. Reference herein to any specific commercial product, process, or service by trade name, trademark, manufacturer, or otherwise does not necessarily constitute or imply its endorsement, recommendation, or favoring by the United States Government or any agency thereof. The views and opinions of authors expressed herein do not necessarily state or reflect those of the United States Government or any agency thereof. 


\section{A. Objectives}

The long-range objective of this research program is the development of more efficacious technetium-99m radiopharmaceuticals for use as imaging agents in diagnostic nuclear medicine. Within this overall goal, we seek to isolate and develop distinct site imaging agents, each of which has properties optimized to provide diagnostic information concerning a given pathological condition. These objectives are being accomplished by the development of analytical techniques which (a) enable complete analysis of radiopharmaceutical preparations so that individual complexes can be characterized with respect to imaging efficacy and (b) enable a radiopharmaceutical to be monitored after injection into a test animal to determine the species that actually accumulates in an organ to provide the image. Administration of the isolated, single most effective imaging complex, rather than a mixture of technetium-containing complexes, will minimize radiation exposure to the patient and maximize diagnostic information available to the clinician.

The specific objectives for this funding period are as follows:

(1) Development of an improved separation technique (capillary electrophoresis, $C E$ ) for the characterization of diphosphonate skeletal imaging agents. $C E$ should be applicable to the analysis of radiopharmaceuticals before injection and of excreted complexes in urine. This technique will be developed primarily with diphosphonate skeletal imaging agents, with which we have considerable experience.

(2) Development of electrochemical and fiber optic sensors for TC and Re imaging agents. These sensors will ultimately be capable of monitoring a specfic chemical state of an imaging agent in vivo after injection into a test animal by implantation in the organ of interest. Sensors for heart imaging agents will be developed first because of their optimum electrochemical and optical properties with respect to sensor requirements.

\section{B. Development of Capillary Electrophoresis as a separation Technique for Nuclear Medicine}

We have achieved our first specific objective, which is to assemble a CE instrument with UV-Visible detection. The complete instrument consists of a high voltage power supply Model 210-30p by Bertan, a high voltage supply controller series HVC by Bertan, a CE absorbance detector CV by ISCO, an integrator HP 3394 by Hewlett Packard and a homemade safety box including the buffer vials, the capillary and the electrodes. In order to test the assembled instrument and to gain experimental experience a series of experiments were performed to separate a mixture of three model compounds: aniline, $p$-phenosulfonic acid ( $p$-PSA), and $m-$ aminobenzoic acid $(m-A B A)$ 
In $C E$ the reproducibility of the electrosmotic flow (EOF) is considered to be one of the greatest problems. To evaluate the EOF from the results obtained the attention was focused on the migration times $t_{m}$, rather than on efficiency or resolution. Initial problems included a steady baseline decay, considerable increase of $t_{m}$ from run to run, and a long analysis time were overcome by lowering the applied voltage, lowering the buffer concentration, changing the detection wavelength, using a buffer of different composition, and performing a rinsing procedure after each run.

Under these conditions a separation of the three-component mixture was achieved within a reasonable abnalysis time of $15 \mathrm{~min}$. However after two or more runs an increase of migration times and a decrease of peak heights (peaks of late eluting compounds disappeared completely). was observed. This phenomenon was probably caused by processes on the capillary wall and was successfully defeated by allowing the capillary to equilibrate at a potential of $\mathrm{U}=10 \mathrm{kV}$ for $600 \mathrm{sec}$ after rinsing.

We are now beginnign to develop conditions for separation of diphosphonate skeletal imaging agents using Re- and Tc-MDP as a model system.

\section{c. Development of sensors for in vivo Monitoring of Radiopharmaceuticals}

In vivo monitoring involves the detection of a compound at a microelectrode (diameter ca. $10 \mu \mathrm{m}$ ) or by a fiber optic probe that has been implanted in a particular tissue or organ of the test animal. Inherent in this approach is the ability to achieve realtime monitoring of the compound in a live animal. Our objective in this project is to develop sensors for in vivo monitoring technetium/rhenium compounds used for imaging the heart. our strategy for development of an in vivo sensor for $99 \mathrm{~m}_{\mathrm{Tc}}$ heart agents is a microelectrode or fiber optic sensor that would reiy on a polymer coating into which the agent would partition and thereby enhance its detection in the biological matrix.

\section{Electrochemical sensors for TC and Re Imaging Agents}

The technique of in vivo voltammetry with implanted microelectrodes offers a good means of monitoring a technetium complex without alterating its chemical nature. The main difficulty with this approach is achieving the selectivity to detect the technetium complex at the required concentration level in the presence of other electroactive species in the organ of interest such as ascorbic acid and uric acid. Both detection limit and selectivity can be improved by taking advantage of the charge and lipophilic properties of the technetium complexes. The electrode surface can be modified with a thin film of polymeric membrane into which the technetium complex preferentially extracts from the aqueous environmenc of the organ tissue, thereby preconcentrating it at the electrode surface as shown schematically in Figure 1. This film 
would likely impede diffusion of non-lipophilic materials, such as ascorbic acid and uric acid, to the electrode surface and thereby diminish their concentration at the surface and, consequently, reduce any interfering signal.

Previous electrochemical studies performed on $\left[M^{I I} D_{2} X_{2}\right]^{\circ}$ where $M$ is TC and $R e, D$ is DMPE, and $X$ is $C l$ or $B r$ in aqueous and aqueous micellar solutions have shown a well-defined cyclic voltammogram that corresponds to the M(III/II) redox couple.<smiles>CP(C)CP(C)C</smiles>

DMIPE, 1,2-bis(dimethylphosphino)ethane

Cyclic voltammetry of $\left[\mathrm{Re}(\mathrm{DMPE})_{3}\right] \mathrm{CF}_{3} \mathrm{SO}_{3}$ in aqueous solution at gold, platinum, glassy carbon, and spectroscopic graphite electrodes at concentration ranges of $10^{-4} \mathrm{M}$ to $10^{-4} \mathrm{M}$ show welldefined voltammograms for a one-electron transfer between the monoand dication forms of the complex. Cyclic voltammetry and chronocoulometry experiments suggest that the complex adsorbs on gold and carbon electrodes, but not on platinum. Thin layer spectroelectrochemistry experiments give a linear Nernst plot with an $\mathrm{n}$ value of one and an $\mathrm{E}^{\circ}$ ' of $-0.006 \mathrm{~V}$ Vs. Ag/AgCl.

Various polymers have been evaluated as preconcentrating films on the electrode surface. of these, the cation exchange polymer Nafion works best. Evaluations of both commercially available Nafion film and Nafion gels have been made at glassy carbon and spectroscopic graphite electrodes. The electrochemistry of the rhenium complex at these Nafion gel modified electrodes is still a single, well-defined redox couple located at $0.062 \mathrm{~V}$. However, peak currents for cyclic voltammetry and differential pulse voltammetry show an enhancement of up to $100 \mathrm{x}$ (compared to bare electrodes) for $10^{-6} \mathrm{M}$ solutions due to partitioning of the complex into the Nafion film. A cyclic voltammogram of the rhenium complex in the polymer film is shown in Figure 2 .

Calibration plots by differential pulse voltammetry show a linear relationship between peak current and concentration of complex in the $10^{-6}$ to $10^{-7} \mathrm{M}$ range with plateauing at higher concentrations due to saturation of the film. partitioning of the [Re(DMPE) $\left.{ }_{3}\right] \mathrm{CF}_{3} \mathrm{SO}_{3}$ complex into disks of Nafion polymer has been monitored by absorbance spectroscopy. Modified Nafion gels 
monitored by absorbance spectroscopy. Modified Nafion gels preconcentrated the rhenium complex more rapidly and to a greater degree than the commercially available film. Thus, Nafion gel is a promising candidate for surface modification of electrodes for the detection of cationic imaging agents.

\section{Fiber Optic sensors}

Several significant milestones have been passed in our efforts to develop the physical optics of a fiber optic absorbance sensor for radiopharmaceuticals. These include [1] the design and fabrication of the interface for the fiber optic absorbance sensor, [2] the refinement of the fiber sensor's distal optics to include a specially-prepared graded-index microlens, and [3] the refinement of the end mirror for the sensor. A general diagram of this sensor type is shown in Figure. 3. In an ultimate clinical or animal experimental setting, we envision a single small instrumentation module which would contain all the needed instrumentation. The sensor would be tethered by any desirable length of fiber optic to this module. Thus, applications involving radioisotopes could be accomplished without unnecessary exposure of any more than the sensor tip to radioactive species.

The sensor configuration has been chosen to be of the single fiber type. This choice obviates the need for high-precision assembly of the sensor distal optics as well as rendering the sensor diameter a minimum. In this regard, we have designed the sensor to fit within standard catheter-sized stainless steel tubings.

As an example of the potential utility of the fiber optic sensor, we show (Figure 4) the absorption spectrum of a non-radioactive heart imaging agent analogue, trans-[Re $\left.\left(\mathrm{p}-\mathrm{SC}_{6} \mathrm{H}_{4} \mathrm{CH}_{3}\right)_{2}(\mathrm{DMPE})_{2}\right]^{+}$in ethanolic solution. The spectrum shown was taken without the benefit of an included preconcentration polymer. Nonetheless, from this and similar results the limit of detection can be estimated and it is about $4 \times 10^{-7} M$ without using a preconcentration polymer. Assuming the use of a preconcentration polymer with an enhancement factor of $100 x$, the probable limit of detection would be then be about $4 \times 10^{-9} \mathrm{M}$ for this rhenium analogue. Investigations with a large number of such fiber optic sensors shows that these sensors dependably report absorbance values in the range of $0.004-1.500$ au which are strictly linear with analyte concentration. The development of an optically transparent preconcentration polymer is in progress. 


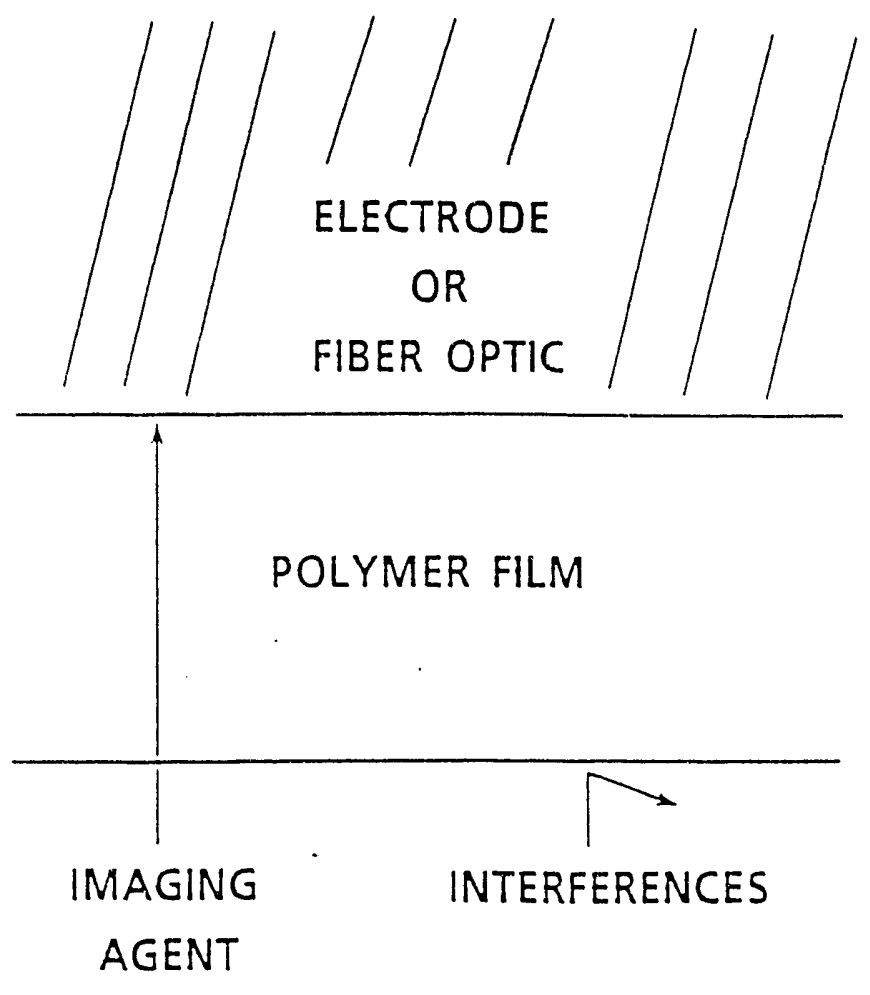

Fig. 1. Diagram of sensor surface.

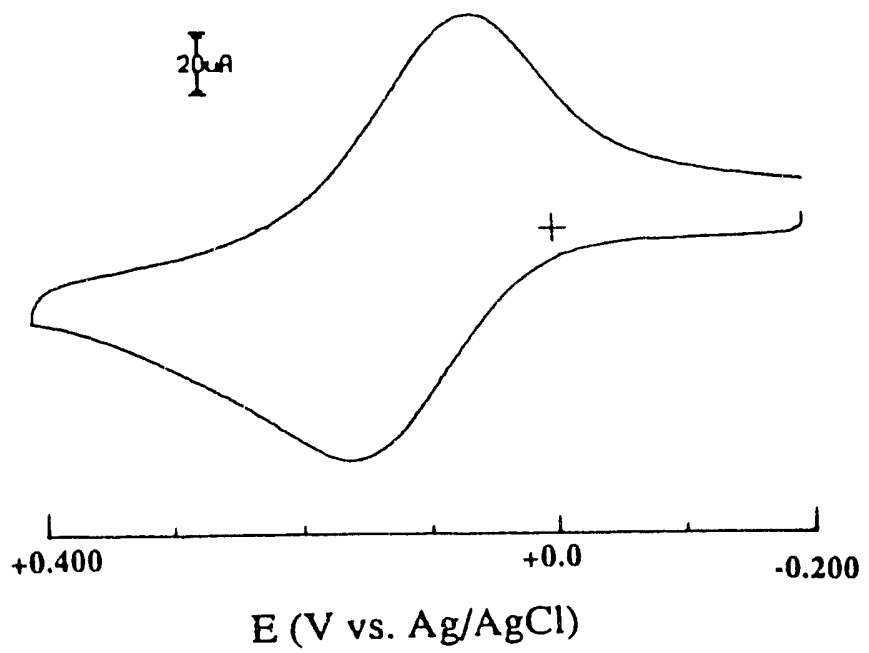

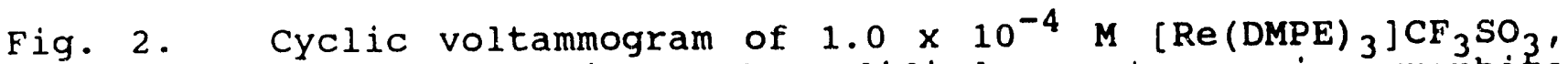
$0.15 \mathrm{M} N \mathrm{NaCl}$ at a Nafion gel modified spectroscopic graphite electrode after immersion in solution for $1 \mathrm{~h}$. Scan rate $100 \mathrm{mV} / \mathrm{s}$, deoxygenated with argon. 


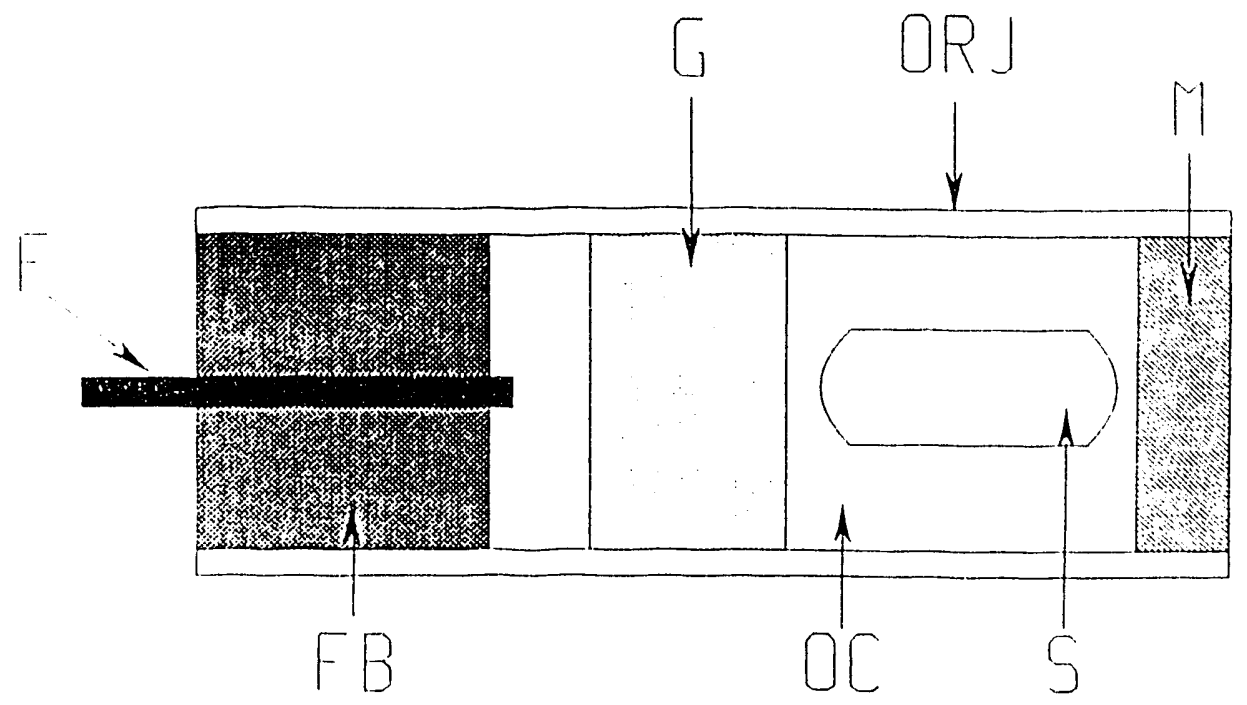

Fig. 3. A general diagram of the fiber optic sensor is shown. The symbols denote: F, fiber optic; FB, fiber optic bushing; G, gradedindex microlens; OC, optical cavity; ORJ, outer rigid jacket (stainless steel tubing); $S$, slit(s) for solution entry to optical cavity; $M$, end mirror.

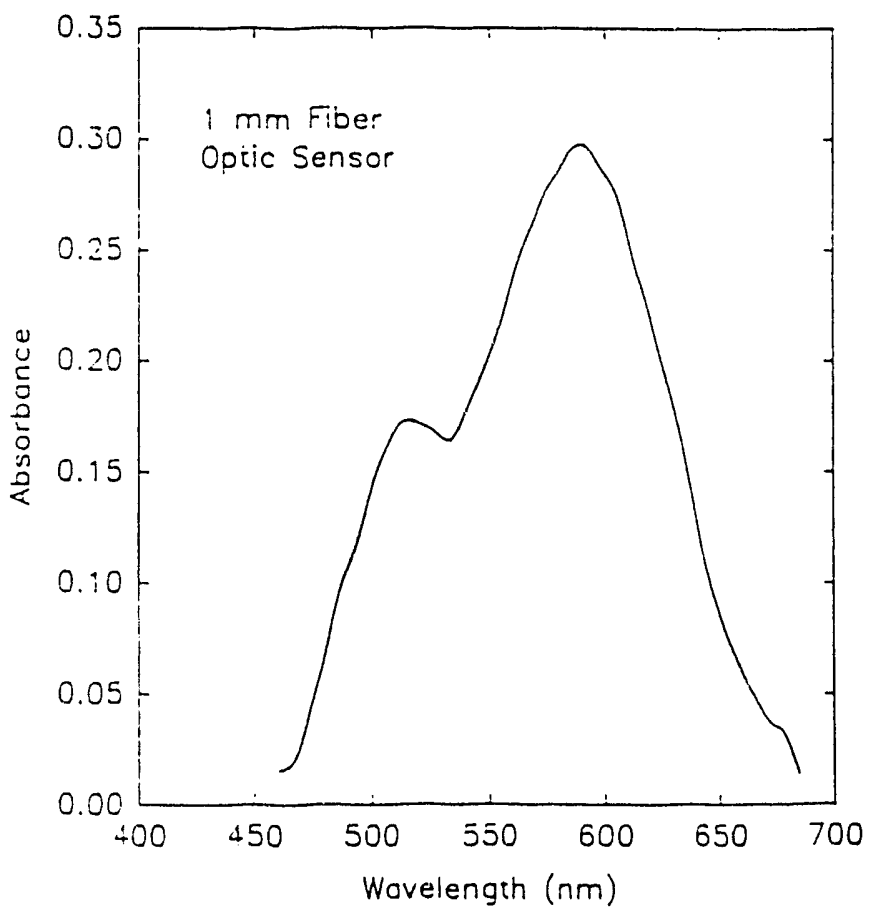

Fig. 4 The absorbance spectrum of trans-[Re $(\mathrm{p}-$ $\left.\mathrm{SC}_{6} \mathrm{H}_{4} \mathrm{CH}_{3}\right)_{2}(\mathrm{DMPE})_{2} \mathrm{PF}_{6}$ in absolute ethanol at $2.4 \mathrm{x} 10^{-5} \mathrm{M}$
concentration is shown. The fiber optic sensor used was 1 mm in diameter and had an optical pathlength of $10.0 \mathrm{~mm}$. 

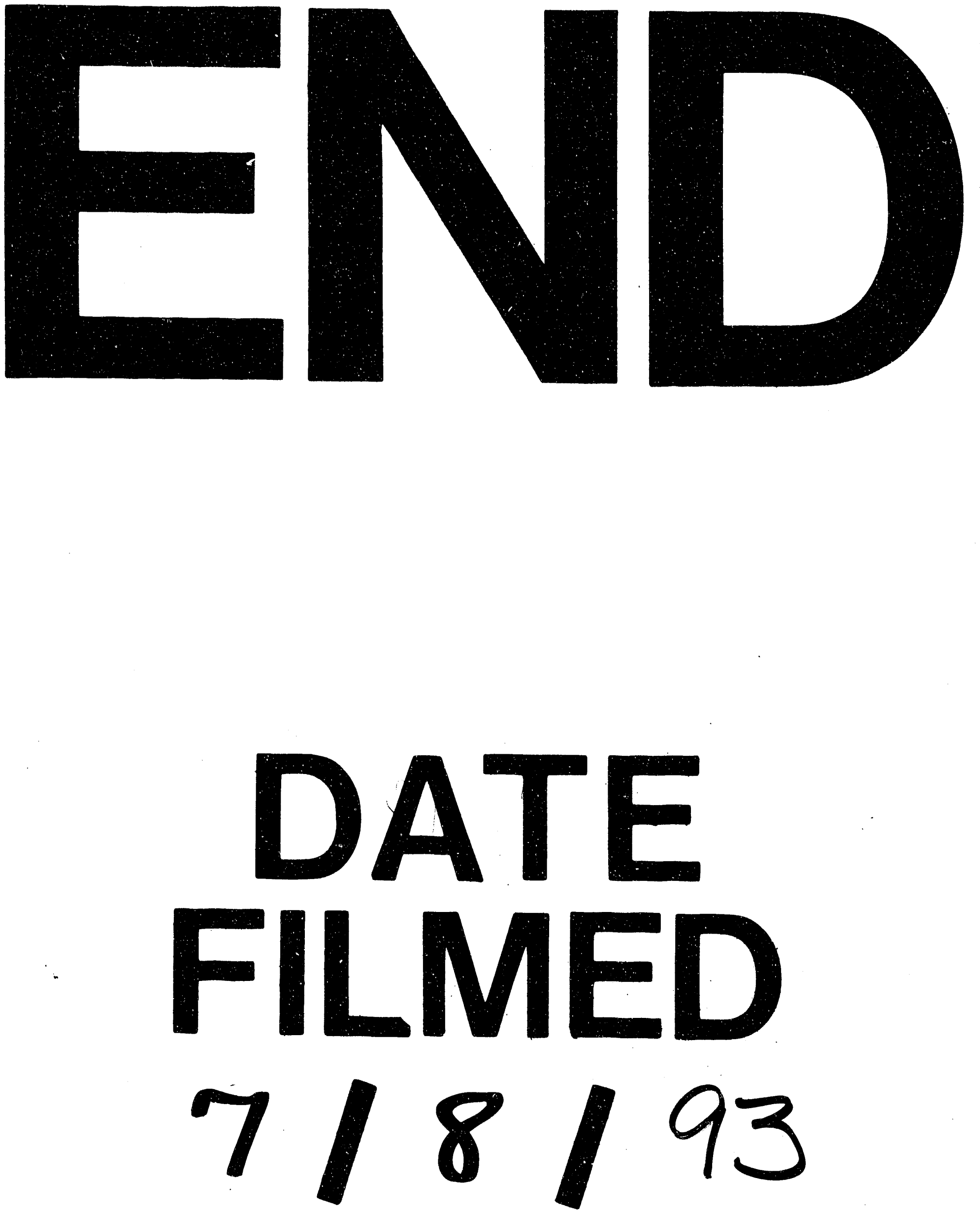
